\title{
Magneto-induced Self-assembling of Conductive Nanowires for Biosensor Applications
}

\author{
Javier Jiménez, Roman Sheparovych, Marcos Pita, Arántzazu Narvaez García, Elena \\ Dominguez, Sergiy Minko*and Evgeny Katz* \\ Department of Chemistry and Biomolecular Science, \\ Clarkson University, Potsdam NY 13699-5810, USA
}

\section{Supporting information}

Functionalization of the cobalt ferrite $\left(\mathrm{CoFe}_{2} \mathrm{O}_{4}\right)$ magnetic nanoparticles with amine and thiol groups: The reaction was carried out at room temperature $\left(23 \pm 2{ }^{\circ} \mathrm{C}\right)$ by mixing in a round-bottomed flask $288 \mathrm{~mL}$ of absolute ethanol, $2 \mathrm{~mL}$ of the cobalt-ferrite ferrofluid (ca. $16 \mathrm{mg} \mathrm{mL}^{-1}$ ) and $12 \mathrm{~mL}$ of $30 \% \mathrm{NH}_{3}$ solution, according to the published procedure. ${ }^{1}$ The silane reactant mixture was added up to a final concentration of $0.5 \%$. Two silane coupling agents were employed, $\gamma$-aminopropyltriethoxysilane (APTES) (Sigma, ACS reagent) and $\gamma$-mercaptopropyltrimethoxysilane (MPTMS) (Sigma, ACS reagent), which append amino and thiol functional groups to the magnetic nanoparticles (NPs) respectively. ${ }^{2}$

Synthesis and attachment of gold nanoparticles (Au-NPs) on amino/thiol-functionalized magnetic nanoparticles: Gold NPs were freshly synthesized in aqueous media by the reduction of $1 \mathrm{mM} \mathrm{HAuCl}_{4}$ (Sigma, ACS Reagent) in $60 \mathrm{mM} \mathrm{NaOH}$ solution with $1 \mathrm{mM}$ tetrakis(hydroxymethyl)phosphonium chloride (THPC) (Fluka). ${ }^{3}$ The reductant was added to the alkaline solution followed by the gold salt under a vigorous stirring. The resulting NPs were filtered with a PTFE $0.2 \mu \mathrm{m}$ pore size filter. A dispersion of the AuNPs (10 mL, $80 \mathrm{nM}$ of particles) was reacted with $10 \mathrm{~mL}$ of amino/thiol-functionalized magnetic NPs $\left(3 \mathrm{mg} \cdot \mathrm{mL}^{-1}\right)$ in order to achieve the attachment of gold-seeds on the magnetic NPs functionalized surface. The obtained gold-seed-modified magnetic NPs were separated and purified by magnetic attraction and extensive rinsing with water. 
Formation of a gold-shell on the magnetic nanoparticles: The gold shell around the magnetic NPs was generated according to a previously published procedure ${ }^{2}$ with its minor modification. $1 \mathrm{~mL}$ of the solution containing $3 \mathrm{mg} \cdot \mathrm{mL}^{-1}$ of the gold-seed-modified magnetic NPs was diluted ten times with Milli-Q water, followed by the addition of 10 $\mu \mathrm{L}$ of $2.5 \mathrm{mM} \mathrm{HAuCl}_{4}$ under vigorous stirring. The gold reduction was carried out by adding $30 \mu \mathrm{L}$ of a freshly prepared $5 \mathrm{mM}$ hydroxylamine hydrochloride $\left(\mathrm{NH}_{2} \mathrm{OH} \cdot \mathrm{HCl}\right)$ solution. ${ }^{4}$ After the gold salt reduction, the NPs were magnetically sedimented, washed and decanted twice with $1 \mathrm{~mL}$ of water to remove reaction by-products. This procedure was repeated six times in order to achieve a complete shell covering.

\section{References:}

1. Wagner, J.; Autenrieth, T.; Hempelmann, R. J. Magnetism Magnetic Mater. 2002, 252, 4-6.

2. Fernández, V.M.; Martín Gago, J.Á.; Pita, M.; Serna, J.C.; Briones, C.; Vaz, C.; Mateo-Martí, E. Spanish patent application (200502269) and PTC application (PTC/ES2006/070134).

3. Duff, D.G.; Baiker, A.; Edwards, P.P. Langmuir 1993, 9, 2301-2309.

4. Lyon, J.L.; Fleming, D.A.; Stone, M.B; Schiffer, P.; Williams, M.E. Nano Lett. 2004, 4, 719-723. 


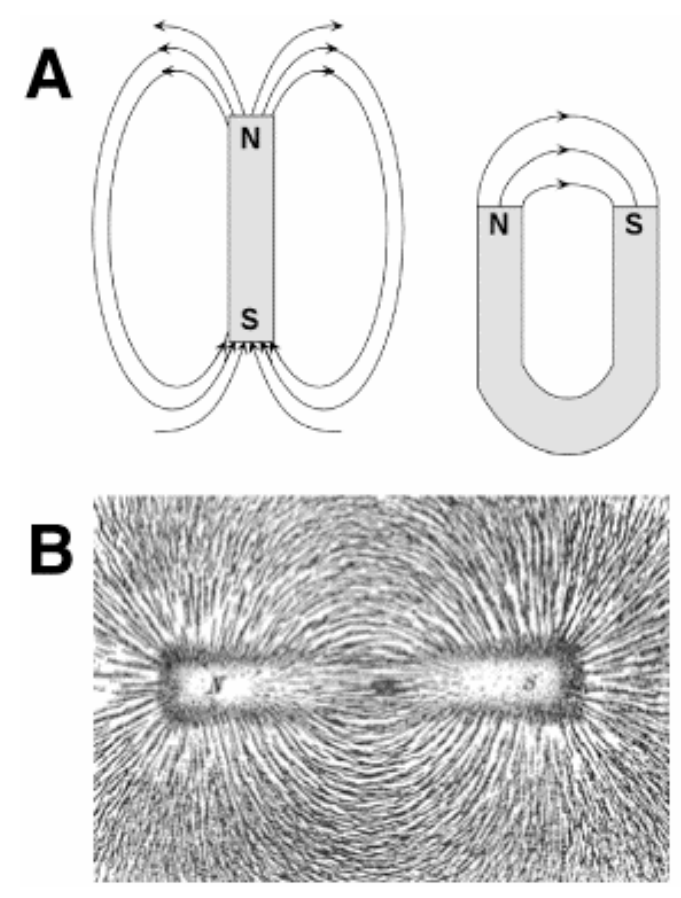

Figure S-1: (A) Schematic representation of the concept of the magnetic field lines (B) Visualization of the magnetic field lines by placing ferromagnetic species in the field. (Note that the present scheme and the picture are given for the illustration only and they do not reflect the field distribution in the present study.)
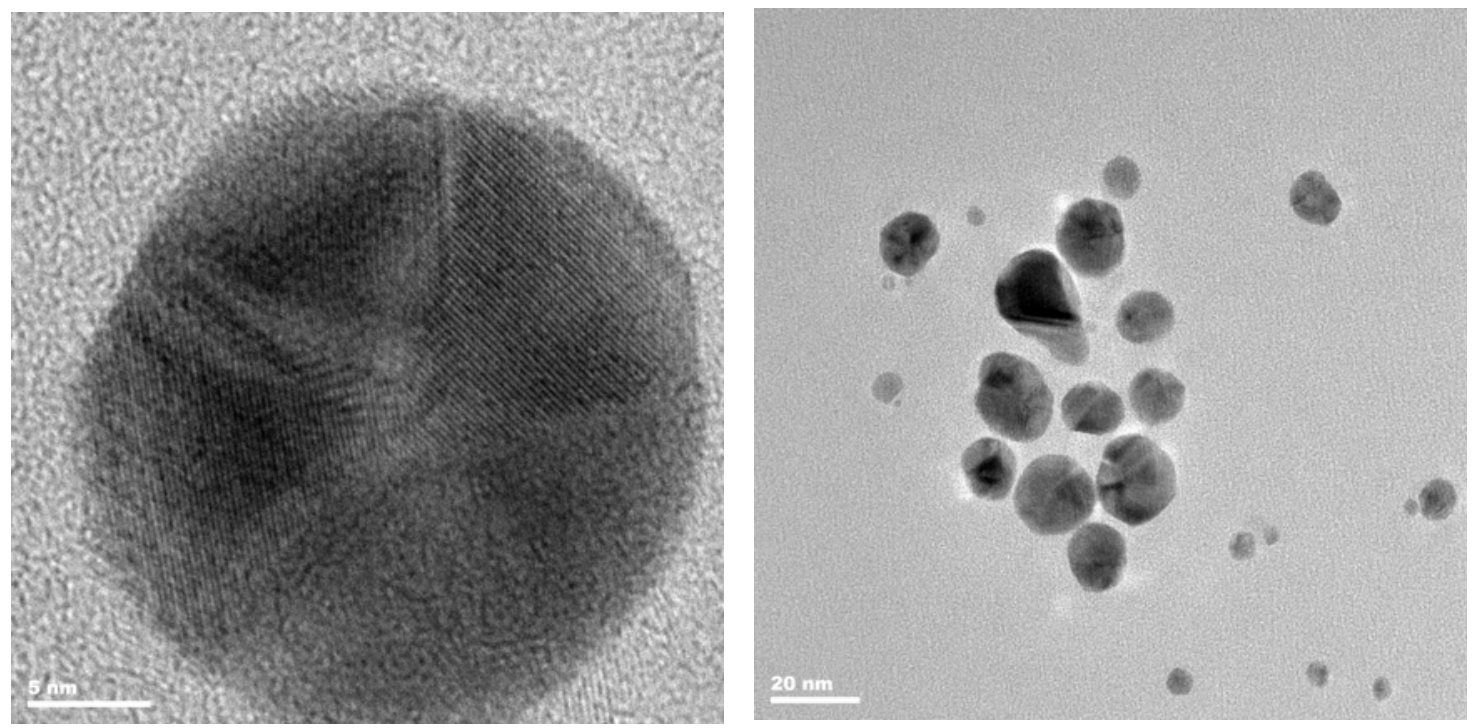

Figure S-2: TEM images of the Au-shell/magnetic core NPs. 


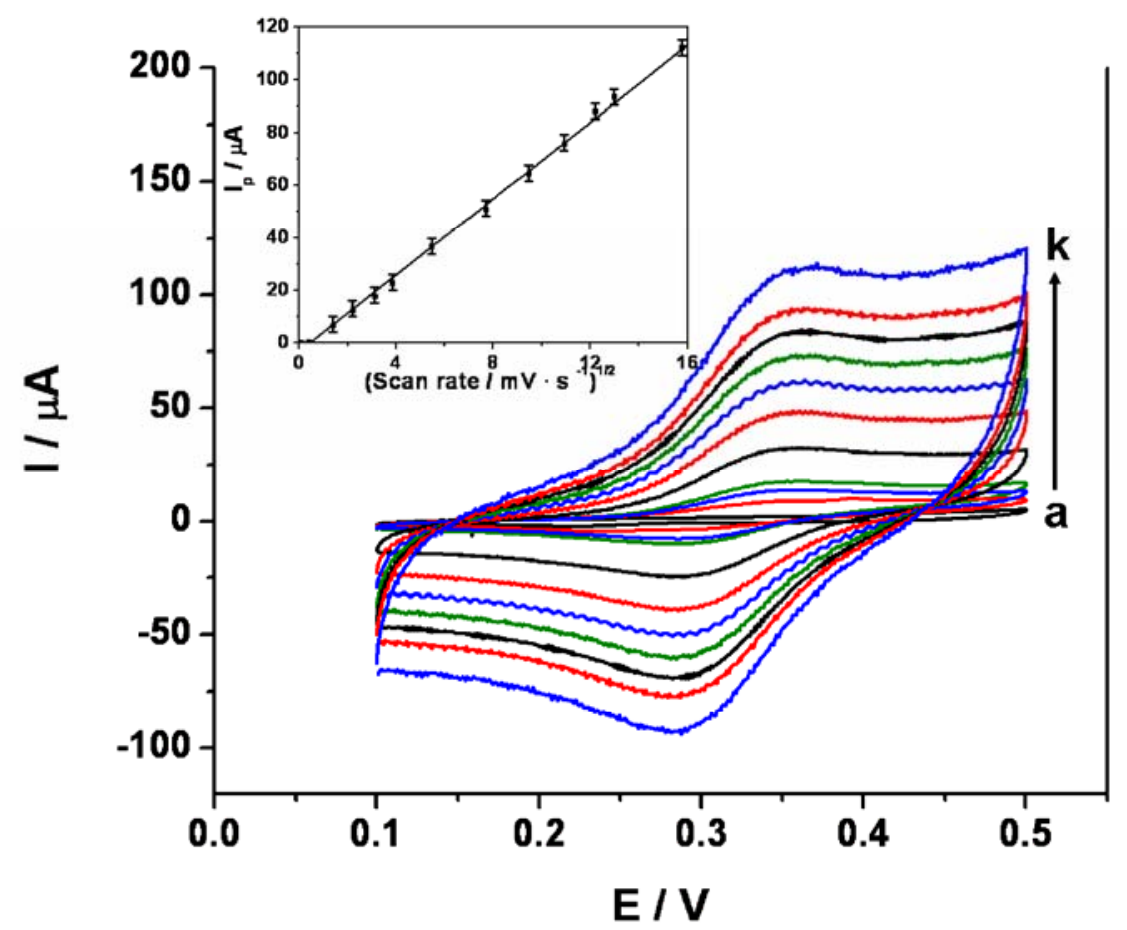

Figure S-3: Cyclic voltammograms for ferrocenemonocarboxylic acid at the bare Au electrode obtained with different potential scan rates: a) 2, b) 5 , c) 10 , d) 15 , e) 25$, f) 50, g) 75, h) 100, i) 125, j) $150, \mathrm{k}) 200 \mathrm{mV} \cdot \mathrm{s}^{-1}$. Inset: Peak current dependence on the square root of the potential scan rate. The experiment was performed using $0.1 \mathrm{mM}$ ferrocenemonocarboxylic acid solution in $0.1 \mathrm{M}$ phosphate buffer, $\mathrm{pH}$ 7.0, under Ar. 


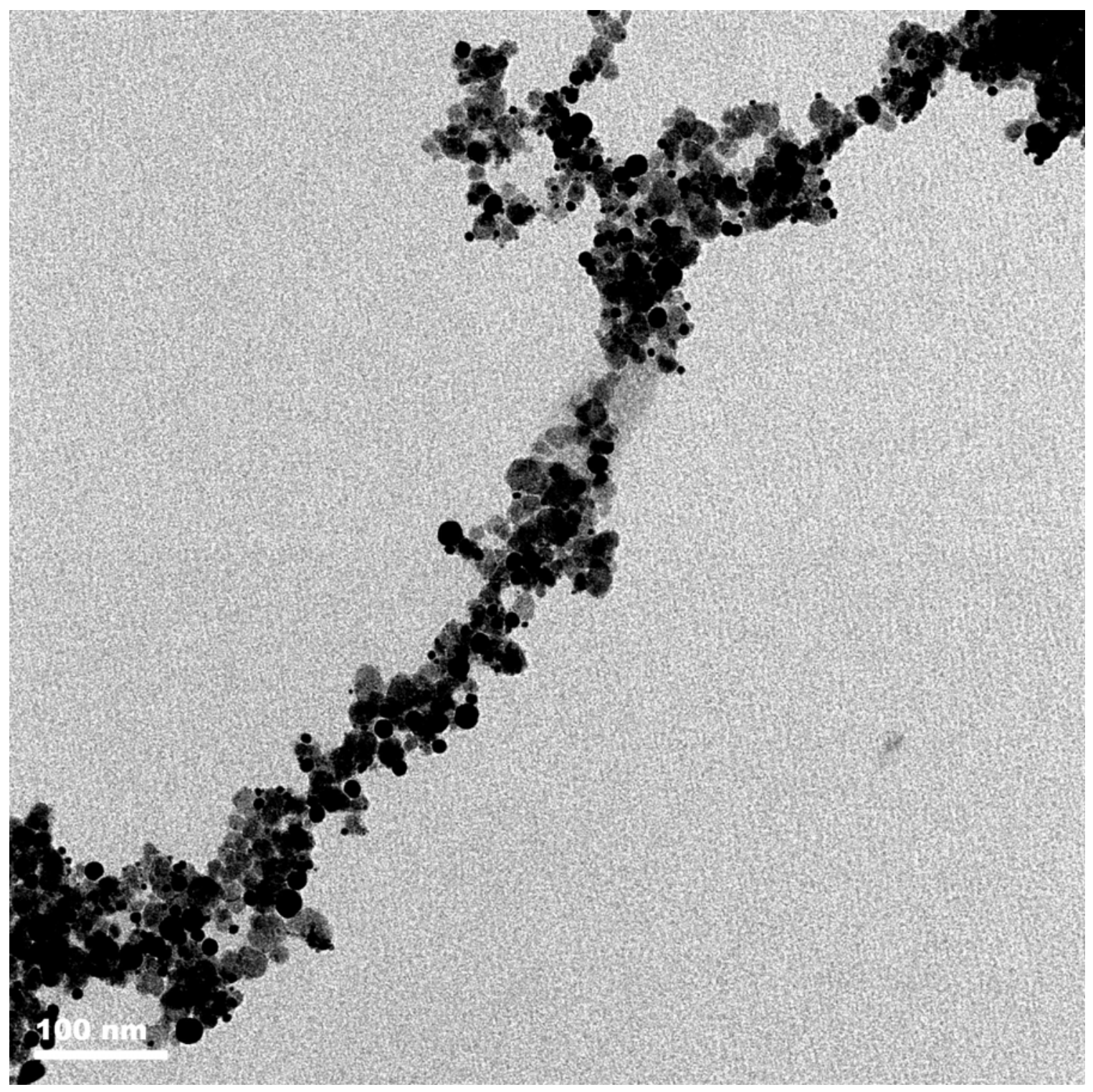

Figure S-4. TEM picture of gold-covered magnetic nanoparticles assembled to form a nanowire. 